\title{
Physical parameters of the Algol system TZ Eridani from simultaneous analysis of Geneva 7-colour light curves $^{\star, \star \star}$
}

\author{
F. Barblan ${ }^{1}$, P. Bartholdi ${ }^{1}$, P. North ${ }^{2}$, G. Burki ${ }^{1}$, and E.C. Olson ${ }^{3}$ \\ 1 Geneva Observatory, CH-1290 Sauverny, Switzerland \\ 2 Institut d'Astronomie de l'Université de Lausanne, CH-1290 Chavannes-des-Bois, Switzerland \\ 3 Astronomy Department, University of Illinois, 1002 W. Green St., Urbana, Illinois 61801, U.S.A.
}

Received March 27; accepted May 11, 1998

\begin{abstract}
Light curves of the semi-detached eclipsing binary system TZ Eridani in the Geneva 7-colour photometric system were analysed using the Wilson-Devinney programme. The physical and orbital parameters have been determined through a self-consistent simultaneous solution of the seven light curves and of the radial velocity curves of both components. The following absolute elements of the components are, for the primary (mass gainer), $M_{1}=1.97 \pm 0.06 M_{\odot}, R_{1}=1.69 \pm$ $0.03 R_{\odot}, M_{\mathrm{bol}_{1}}=2.36 \pm 0.09, T_{\mathrm{eff}_{1}}=7770 \pm 100 \mathrm{~K}$, and for the secondary (mass loser), $M_{2}=0.37 \pm 0.01 M_{\odot}, R_{2}=$ $2.60 \pm 0.04 R_{\odot}, M_{\mathrm{bol}_{2}}=3.74 \pm 0.13, T_{\mathrm{eff}_{2}}=4570 \pm 100 \mathrm{~K}$. The semi-major axis $A$ of the relative orbit is $10.57 \pm$ $0.16 R_{\odot}$. The regular increase of the period is described. The spectral type of the components are A5/6 V (primary) and K0/1 III. The secondary has exhibited a long-term luminosity increase of about 0.06 in $V$ between Dec. 1983 and Dec. 1996. The distance to TZ Eri is evaluated to $270 \pm 12$ pc.
\end{abstract}

Key words: stars: individual: TZ Eridani — binaries: eclipsing — stars: fundamental parameters

\section{Introduction}

TZ Eridani (BD -6 $\left.6^{\circ} 880\right)$ is an Algol-type EA eclipsing binary of short period, $P=2.606$ days. The total primary eclipse is $2.5 \mathrm{mag}$ deep in the $V$ band. The estimation of the spectral type of the two components is $\mathrm{F}$ (Cannon

Send offprint requests to: F. Barblan

* Based on observations made at the European Southern Observatory (La Silla, Chile) and at the Mount Laguna Observatory (U.S.A.)

$\star \star$ Table 1 is only available in electronic form at the CDS via anonymous ftp to cdsarc.u-strasbg.fr (130.79.128.5) or via http://cdsweb.u-strasbg.fr/Abstract.html
1934), F8 (Brancewicz \& Dvorak 1980) for the primary, and K0IV (Kaitchuck \& Park 1988), K0 (Yoon et al. 1994) for the secondary. However, as shown in this paper, the most probable spectral types are $\mathrm{A} 5 / 6 \mathrm{~V}$ for the primary and K0/1 III for the secondary.

A first estimate of the physical parameters of the components is given by Brancewicz \& Dvorak (1980), in their "Catalogue of Parameters for Eclipsing Binaries", in particular $R_{1}=1.51 R_{\odot}$ (primary component) and $R_{2}=$ $1.92 R_{\odot}$. Recall that these values have not been obtained by a simultaneous treatment of the complete light and radial velocity curves. It is the aim of this paper to perform such a new analysis, by using the method and the computer programme of Wilson \& Devinney (1971).

No complete light or radial velocity curves have been yet published for TZ Eri. For that reason, this star was measured intensively in the 7-colour Geneva photometric system (Golay 1980; Rufener 1988) using the Swiss telescope at La Silla (European Southern Observatory, Chile) equipped with the two-channel aperture photometer P7 (Burnet \& Rufener 1979). Moreover, the radial velocity curve of the primary component has been determined with the spectrovelocimeter CORAVEL installed on the $1.54 \mathrm{~m}$ Danish telescope at La Silla, and with the Illinois Cassegrain ("white") spectrograph attached to the $1 \mathrm{~m}$ Illinois reflector at Mount Laguna Observatory. In addition, one spectrum of the secondary component has been obtained by using the NTT $3.5 \mathrm{~m}$ telescope at La Silla, allowing the determination of the masses of the two components of TZ Eri.

TZ Eri was discovered to have a disk (Kaitchuck \& Honeycutt 1982) on the basis of emission in $\mathrm{H}_{\beta}$ and $\mathrm{H}_{\gamma}$ lines observed during the eclipse. Kaitchuck \& Park (1988) showed that this disk belongs to the class of the transient accretion disks which are produced by a collision of the gas stream with the mass-gaining star (primary component). The radius of the disk was measured by the duration of the presence of $\mathrm{H}_{\beta}$ line in emission after the start of the 
primary eclipse (second contact) or before the end of the same eclipse (third contact). The disk extension is variable from one eclipse to another, and in most cases between the trailing and leading sides. During the 12 eclipses studied, the disk extension $r / R_{1}$ varied from 1.0 to 1.64 .

In this paper, the variation of the period of TZ Eri will be analysed, the variability of the components will be examined, and the physical parameters of the two components will be determined from the analysis of the light and radial velocity curves.

\section{Period}

The orbital period listed in the GCVS (Kholopov 1985) is $P=2.6060653$ days. However, we are dealing here with a semi-detached system which, by definition, has a variable period due to mass transfer. During most photometric observations done in 1984-1987 by Dr. Zdenek Kviz, the period remained fairly constant, since it was possible to obtain a well-defined lightcurve with $P=$ 2.6061082 days. This value was obtained using the reciprocal $\theta_{1}$ test of Renson (1978) - see also Manfroid et al. (1991) - which had been defined above all for Ap stars (which vary with small amplitudes) but proved very efficient for a precise determination of the period of eclipsing binaries. The ephemeris we have adopted is:

$$
\begin{aligned}
\operatorname{HJD}(\text { Min I })= & (2446109.6922 \pm 0.0010) \\
+ & (2.6061082 \pm 0.0000020) \times E .
\end{aligned}
$$

The orbital period has varied, as shown by the times of minima registered over decades by amateur astronomers. The $\mathrm{O}-\mathrm{C}$ values so obtained, and published in the BSAG Bulletin (e.g. Locher 1997) are shown in Fig. 1 together with a parabolic fit which gives:

$$
\begin{aligned}
\mathrm{O}-\mathrm{C}= & -(0.0050 \pm 0.0014) \\
& -(1.008 \pm 0.366) 10^{-6}\left(\mathrm{HJD}-t_{0}\right) \\
& +\left(2.433 \pm 0.14310^{-9}\left(\mathrm{HJD}-t_{0}\right)^{2}\right.
\end{aligned}
$$

where $t_{0}=2446109.6922$. The typical error on the epochs of primary minima in Fig. 1 is a few minutes (0.002$0.005 \mathrm{~d})$. Therefore the fit only represents a mean trend, upon which are superposed sudden period changes that cannot be accounted for by measurement errors (see especially the very steep rise just before JD 2450000 ).

Although the period changes are interesting by themselves, they are rather a nuisance in our context, because the radial-velocity measurements, which were made recently, have to be put in phase with the photometric ones, which are much older. For this reason we have not used the above formula for $\mathrm{O}-\mathrm{C}$, but we have simply used additional photometric measurements kindly made by Marc Künzli in November 1996 with the same equipment. He has made 36 new multicolour measurements, several of which during the primary minimum. Using the code EBOP16 (Etzel 1989) and the adopted ephemeris,

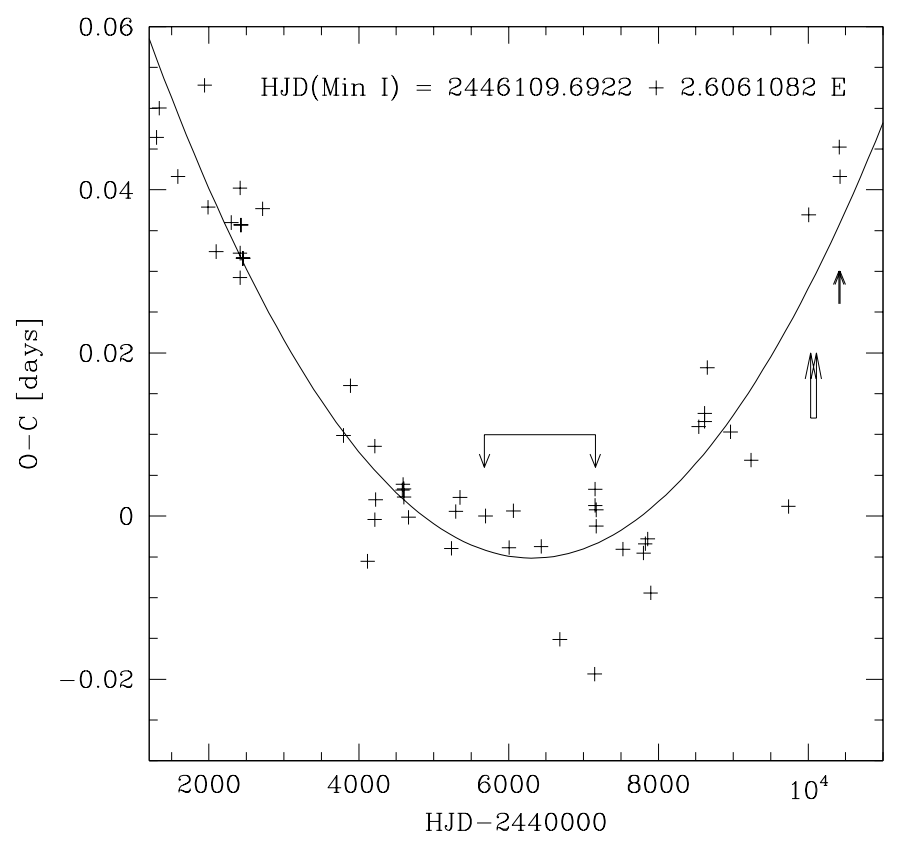

Fig. 1. O-C diagram of TZ Eri from observations made by amateurs and published in the BSAG Bulletin (two points are defined by Geneva photometry). A parabolic fit is superposed, showing the regular increase in the period. Short arrows define the intervals of photometric measurements; long arrows define the interval of radial-velocity observations

we adjusted the $\Delta \theta$ parameter (phase correction for the position of the primary minimum) for these recent data, as well as for the old data alone. The difference is:

$\Delta \phi=-0.01734 \pm 0.00020$

and represents the phase correction to be applied to the 1996 data, to fit them into the adopted ephemeris. Although the $V_{\mathrm{r}}$ observations have been made one year earlier than the new photometric ones, we neglect the slight period change that may have occurred in between, compared to the change that has taken place between the old photometric measurements and the $V_{\mathrm{r}}$ ones. Therefore, the above phase shift was applied as such to the $V_{\mathrm{r}}$ data.

\section{Photometric data and variability of the components}

Geneva 7-colour photometric measurements of TZ Eridani were obtained from Dec. 10, 1983 to Dec. 10, 1996, using the Swiss $70 \mathrm{~cm}$ telescope at the European Southern Observatory (ESO), La Silla, Chile. During this period, 429 measurements of weight $q \geq 1$ have been obtained (see Rufener 1988, for the definition of the weight $q$ ). These data are listed in Table 1, including the 36 additional photometric measurements obtained in Nov.-Dec. 1996 (see Sect. 2). 
The magnitudes in each of the seven filters are obtained from the visual magnitude $V$ and the six colour indices in the following manner:

$i=V-[V-B]+[i-B]$

with $i$ representing one of the seven filters $U, B, V, B_{1}$, $B_{2}, V_{1}, G$. Remember that the Geneva $[U-B]$ and $[B-V]$ indices are not normalized to zero for an A0V star as it is the case for the Johnson $U B V$ indices. It is possible to calculate the magnitude of the primary (mass gainer) by subtracting the flux of the secondary (mass loser), at the bottom of the primary eclipse, from the flux of the both components measured together (outside the eclipses). This calculation has been made for each of the seven Geneva magnitudes. In order to minimize the effects of a possible long-term variability (see the end of this section), only the data obtained in December 1983 and January 1984 have been used. The results are given in Table 2 .

It is interesting to compare the observed uncertainties with the mean precision of the measurements made in Geneva photometry. Rufener (1988, Fig. 2) has shown the shape of the mean relation $\sigma_{V}$ vs. $V$ obtained for the non-variable stars, in particular the progressive increase of $\sigma_{V}$ with increasing $V$, for stars fainter than $V \simeq 9$. The same relation can be applied to the seven Geneva magnitudes. Figure 2 of this paper shows a new calculation of this relation, based on the up to date version of our photometric database. On the same figure are plotted the observed values for TZ Eri given in Table 2. A correction has been applied to the uncertainties of the magnitudes for the secondary, because the measurements obtained during the totality of the primary eclipse had shorter integration time than the other ones (4 minutes instead of 12 minutes). The conclusion is that the uncertainties on the measurements of TZ Eri are in agreement with the expected precision. Thus, the components do not exhibit a short-term variability, i.e on a time-scale shorter than about 20 orbital periods.

Table 2. The seven Geneva apparent magnitudes of TZ Eri (measures of December 1983 and January 1984)

\begin{tabular}{lrcr}
\hline Mag. & $\begin{array}{r}\text { TZ Eri A+B } \\
\text { Observed }\end{array}$ & $\begin{array}{c}\text { Secondary } \\
\text { Observed }\end{array}$ & $\begin{array}{r}\text { Primary } \\
\text { Calculated }\end{array}$ \\
\hline$U$ & $10.540 \pm 0.013$ & $14.87 \pm 0.17$ & $10.560 \pm 0.016$ \\
$B 1$ & $9.987 \pm 0.009$ & $13.980 \pm 0.050$ & $10.015 \pm 0.011$ \\
$B$ & $9.011 \pm 0.009$ & $12.584 \pm 0.031$ & $9.052 \pm 0.010$ \\
$B 2$ & $10.409 \pm 0.007$ & $13.670 \pm 0.035$ & $10.464 \pm 0.009$ \\
$V 1$ & $10.299 \pm 0.011$ & $12.809 \pm 0.021$ & $10.406 \pm 0.014$ \\
$V$ & $9.576 \pm 0.010$ & $12.067 \pm 0.019$ & $9.691 \pm 0.013$ \\
$G$ & $10.679 \pm 0.016$ & $12.960 \pm 0.018$ & $10.821 \pm 0.021$ \\
\hline
\end{tabular}

The long-term photometric behaviour of both components has been analysed by comparing our photometric data obtained at 4 epochs, corresponding to the intensive monitoring of the eclipses: Dec. 1983 to Jan. 1984, Jan.

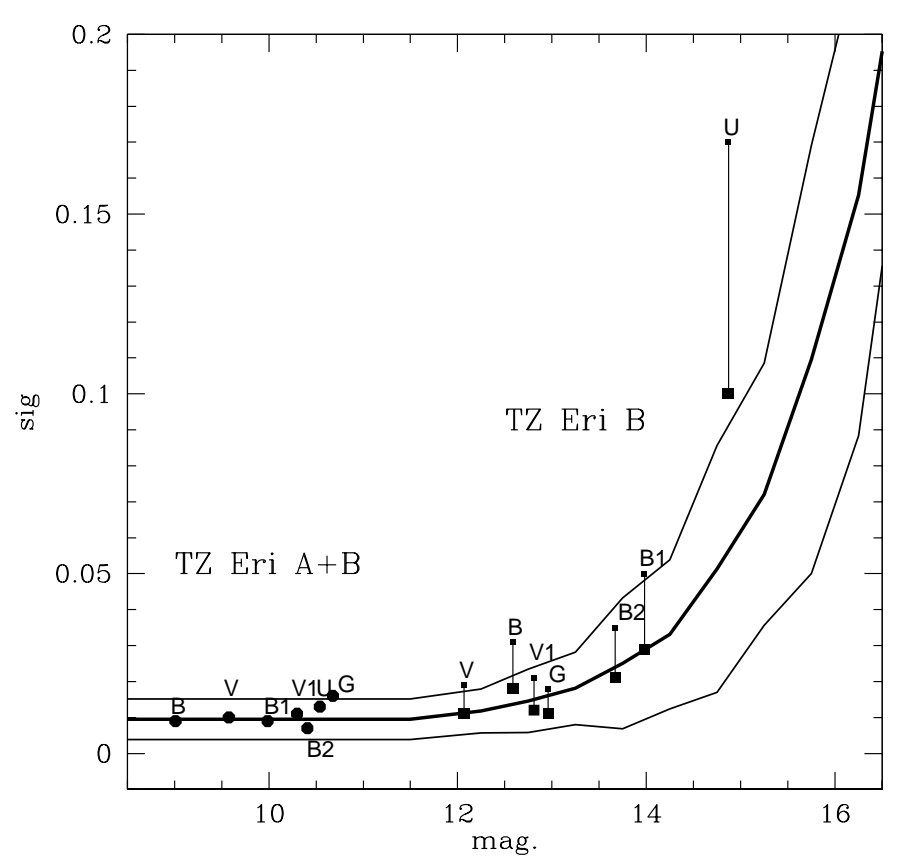

Fig. 2. Variation of the mean precision $\sigma$ with the magnitude, in the case of Geneva photometric measurements. The solid thick line refers to the mean value and the solid thin lines to the 1 s.d. level. Dots and small squares concern respectively the seven magnitudes of TZ Eri A+B outside eclipses and of the secondary (during the totality of the primary eclipse). Big squares represent the estimated values of $\sigma$ for the secondary which ought to have resulted from a "normal" integration time of the measurements, i.e. 12 minutes (normal) instead of 4 minutes (during the primary eclipse)

1985, Nov. 1987 and Nov.-Dec. 1996. Table 3 gives the mean values of $V,[B-V]$ and $[U-B]$ at each of these epochs for the both components. It appears that:

1. The secondary exhibited a long-term luminosity increase $(0.06$ in $V)$ between Dec. 1983 and Dec. 1996. The colour variations are large, especially in $[U-B]$, but not significant due to the large standard deviation.

2. The primary did not show any long-term variation in magnitude or in colours.

The observed variations of TZ Eri secondary are similar to those studied by Olson \& Etzel (1993) in six cool subgiant secondaries of totally eclipsing Algol systems. They noted that the fluctuations increase with decreasing orbital period, or with increasing rotational velocity, suggesting that rotationally induced magnetic activity could be the origin of these brightness variations.

In order to minimize the effects of the long-term variation of the secondary luminosity, only the data obtained before HJD 2447200 (Feb. 1988) have been used for the eclipse analysis (see Sect. 6). 
Table 3. Long-term behaviour of the components of TZ Eri in $V$ magnitude and Geneva colours $[B-V]$ and $[U-B]$. Only the variation of the secondary in $V$ is significant

\begin{tabular}{|l|rrr|rrr|}
\hline & \multicolumn{3}{|c|}{ Secondary (mass loser) } & \multicolumn{3}{|c|}{ Primary (mass gainer) } \\
& $V$ & {$[B-V]$} & {$[U-B]$} & $V$ & {$[B-V]$} & {$[U-B]$} \\
\hline Dec. 1983 & 12.067 & 0.523 & 2.281 & 9.691 & -0.639 & 1.508 \\
& \pm 0.019 & \pm 0.046 & \pm 0.175 & \pm 0.013 & \pm 0.023 & \pm 0.026 \\
Jan. 1985 & 12.045 & 0.466 & 2.184 & 9.691 & -0.639 & 1.561 \\
Nov. 1987 & 12.035 & 0.453 & 1.990 & 9.692 & -0.637 & 1.561 \\
Dec. 1996 & 12.007 & 0.477 & 2.189 & 9.695 & -0.635 & 1.547 \\
\hline
\end{tabular}

\section{Classification of the components and interstellar extinction}

A photometric classification of the components of TZ Eri can be obtained because the primary eclipse is total, by using various calibrations based on the colour indices and reddening free parameters of the Geneva photometric system.

From the magnitudes in Table 2, the various Geneva colour indices and reddening free parameters $d, \Delta$ and $g$ (see Golay 1980) have been calculated. Then, the technique of the photometric boxes (Golay et al. 1969; Nicolet 1981a) has been applied to determine the intrinsic characteristics of the primary component. Recall that this technique is specific to the Geneva photometric system and depends on the homogeneity and precision of its measurements. The assumption in this technique is that the properties of the stars lying within a specified photometric neighbourhood (see Nicolet 1994) can be equated with each other, provided that the radius of the photometric box is small enough. We applied the technique to the parameters $d, \Delta$ and $g$ of the primary and found in the entire photometric database 40 twin stars, i.e. stars having very similar values of the 3 parameters, the radius of the photometric box having been chosen equal to $0.015 \mathrm{mag}$. It is assumed that these stars are intrinsically similar to the primary of TZ Eri. The same method was applied to the classification of the components of the eclipsing RS CVntype system RZ Eridani (Burki et al. 1992).

It is especially noteworthy that 6 of these twin stars belong to open clusters: $\alpha$ Per, Hyades, Praesepe, Pleiades (2 stars) and NGC 6405. This allowed to determine unanbiguously the intrinsic colours of these stars, since these clusters have well determined distances and interstellar extinctions. By using the values given by Nicolet (1981b), we derived the intrinsic colours of the twin stars, and thus also of the primary of TZ Eri, in particular $[B 2-V 1]_{0}=$ $0.011 \pm 0.016$. According to the relations between spectral types and intrinsic Geneva colours or parameters by Hauck (1994) and to the calibration of Geneva photometry based on Kurucz's atmosphere models by Künzli et al. (1997), the estimated spectral type of the primary is $\mathrm{A} 5 / 6 \mathrm{~V}$, its effective temperature is $7770 \pm 100 \mathrm{~K}$, its gravity is $\log g=4.40 \pm 0.07$ and its metallicity is
$[M / H]=0.13 \pm 0.09$. On the other hand, the colour excess is $E[B 2-V 1]=0.047 \pm 0.017$.

For the secondary, we have calculated the intrinsic colours by using the measured values (see Table 1) and the colour excesses obtained for the primary. We obtained in particular $[B 2-V 1]_{0}=0.756$, an estimated spectral type of K0/1 III and an effective temperature of $4520 \mathrm{~K}$.

\section{Radial velocity curves}

The primary's radial velocity curve has been measured with the CORAVEL scanner (Baranne et al. 1979) in December-January 1995-1996. The cross-correlation dip is rather wide and shallow due to the fast axial rotation, so that the scatter of the residuals around the fitted curve is rather large (several $\mathrm{km} \mathrm{s}^{-1}$ ). The secondary component remained invisible to CORAVEL, due to its low luminosity in the blue. About one year later, 14 new observations have been done by E.C. Olson using the Illinois Cassegrain ("white") spectrograph: $38 \mathrm{~cm}$ focal length camera, 831 line $\mathrm{mm}^{-1}$ grating, Texas Instruments $800 \times 800$ CCD detector cooled with liquid nitrogen; the CCD was operated by a Photometric Ltd. controller; spectral resolution in the first order is $0.04 \AA$ per pixel; reductions were done with IRAF.

There has been a problem in fixing the zero-point of the radial velocities obtained with this instrument, so that we fitted an orbit separately to the corresponding data to obtain the apparent systemic velocity. Then we applied to these $V_{\mathrm{r}}$ values a uniform shift equal to the difference between this apparent systemic velocity and that obtained with CORAVEL measurements alone. Indeed, we are confident in the CORAVEL $V_{\mathrm{r}}$ scale, because several standard stars have been observed each night and the small instrumental drifts $\left( \pm 1 \mathrm{~km} \mathrm{~s}^{-1}\right.$ at most) are well controlled. Therefore, the uncertainties quoted in Table 5 may be slightly optimistic, because they refer to an orbital solution which assumed a perfect correction to Olson's radial velocities.

In order to see the secondary star's spectrum and to obtain the mass ratio of the components, we asked Dr. Didier Raboud to observe TZ Eri in the vicinity of a quadrature with the NTT telescope at ESO. He could indeed take one spectrum, with an exposure time of $10 \mathrm{~min}$, 


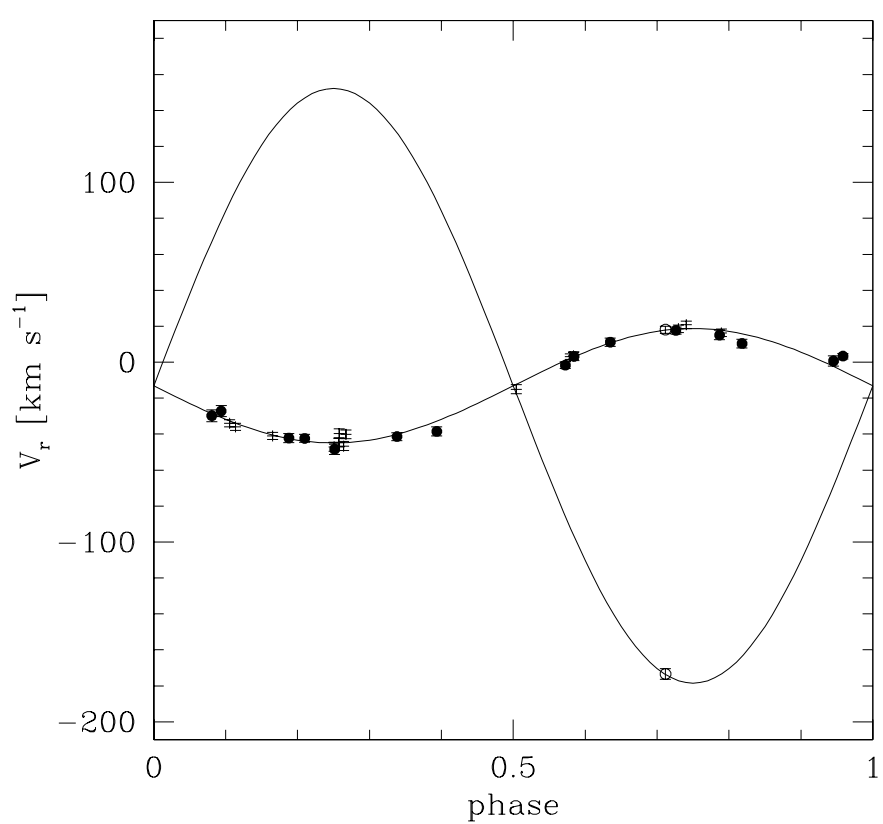

Fig. 3. Radial velocity curve of $\mathrm{TZ}$ Eridani. The black dots (CORAVEL observations) and the plus signs (Olson's observations) represent the primary component, while the open dot represents the secondary. The phases are those of Table 4

on 11th November 1995, using the EMMI spectrograph in the REMD mode, with Grism \#5, Grating \#10 and a slit measuring $1^{\prime \prime} \times 6^{\prime \prime}$; in this configuration, the resolving power is $R=28000$ and the wavelengths range between 4013 and $6606 \AA$. The detector was CCD \#36 (ESO numerotation), a thin, back-illuminated Tektronix TK2048EB chip with $24 \times 24 \mu \mathrm{m}$ pixels. The spectrum has been reduced at Geneva Observatory by Mr. Michel Studer, using the TACOs software developed by Dr. Didier Queloz for the ELODIE spectrograph at Observatoire de Haute-Provence. The radial velocities were obtained by cross-correlation between the observed spectrum and a binary mask optimized for F0-type stars, which yielded two dips, one for each component. Thanks to the long wavelength interval extending well into the red, the cool companion is easily seen in the correlation function. The dips are only $3.6 \%$ and $1.1 \%$ deep for the primary and secondary respectively, but the $\mathrm{S} / \mathrm{N}$ ratio of the correlation function is better than 500. A K0-type mask was tried as well and yielded the same result, but with no improvement.

The journal of the radial velocity observations is given in Table 4 and the radial velocity curve is shown in Fig. 3. Additional spectra would of course be welcome to complete the secondary's $V_{\mathrm{r}}$ curve, but the single point we have suffices to constrain the mass ratio to a precision of about $7 \%(q=0.193 \pm 0.013)$. The orbital elements are given in Table 5 .
Table 4. Journal of the radial velocity observations of TZ Eri. The phases are computed from the ephemeris given by Eq. (1) and corrected for period change according to Eq. (3)

\begin{tabular}{|c|c|c|c|c|}
\hline $\begin{array}{c}\text { HJD } \\
-2400000\end{array}$ & comp. & $\begin{array}{c}V_{\mathrm{r}} \pm \sigma \\
{\left[\mathrm{km} \mathrm{s}^{-1}\right]}\end{array}$ & $\begin{array}{l}\text { Instrument } \\
\text { or observer }\end{array}$ & phase \\
\hline 50033.785 & $\begin{array}{l}\mathrm{A} \\
\mathrm{B}\end{array}$ & $\begin{array}{rl}18.08 & 2 . \\
-173.403 .\end{array}$ & EMMI & 0.712 \\
\hline 50039.606 & A & 0.672 .87 & Coravel & 0.945 \\
\hline 50042.599 & A & -27.193 .08 & & 0.094 \\
\hline 50083.578 & A & 10.322 .50 & & 0.818 \\
\hline 50084.542 & A & -42.172 .55 & & 0.188 \\
\hline 50084.708 & A & -48.402 .89 & & 0.252 \\
\hline 50085.544 & A & -1.721 .87 & & 0.572 \\
\hline 50085.707 & A & 11.172 .30 & & 0.635 \\
\hline 50086.550 & A & 3.381 .56 & & 0.958 \\
\hline 50087.540 & A & -41.392 .17 & & 0.338 \\
\hline 50087.684 & A & -38.532 .46 & & 0.394 \\
\hline 50088.551 & A & 17.601 .96 & & 0.726 \\
\hline 50088.709 & A & 15.042 .63 & & 0.787 \\
\hline 50097.630 & A & -42.392 .23 & & 0.210 \\
\hline 50098.606 & A & 3.242 .37 & & 0.584 \\
\hline 50110.324 & A & -29.783 .29 & & 0.081 \\
\hline 50407.863 & A & $-47.05 \quad 2.5$ & E.C. Olson & 0.251 \\
\hline 50407.880 & A & -44.752 .5 & & 0.257 \\
\hline 50407.897 & A & -46.652 .5 & & 0.264 \\
\hline 50408.730 & A & 3.452 .0 & & 0.583 \\
\hline 50411.717 & A & 18.552 .0 & & 0.729 \\
\hline 50411.745 & A & 20.752 .0 & & 0.740 \\
\hline 50411.872 & A & 16.352 .0 & & 0.789 \\
\hline 50412.697 & A & -34.052 .0 & & 0.106 \\
\hline 50412.719 & A & -35.952 .0 & & 0.114 \\
\hline 50412.853 & A & -40.952 .0 & & 0.165 \\
\hline 50413.735 & A & -15.052 .5 & & 0.504 \\
\hline 50413.931 & A & 3.151 .5 & & 0.579 \\
\hline 50415.700 & A & -39.652 .5 & & 0.258 \\
\hline 50415.725 & A & -40.152 .5 & & 0.267 \\
\hline
\end{tabular}

\section{Rotation and metallicity}

The rotational velocity of the primary deduced from the standard calibration of the width of the correlation dip measured with CoRAVEL (Benz \& Mayor 1984), is

$v \sin i=38.1 \pm 2.2 \mathrm{~km} \mathrm{~s}^{-1}$

which is slightly faster than the synchronous rotation value, since with a radius $R=1.70 R_{\odot}$ (see Sect. 6 ), the expected equatorial velocity of the primary is $33.0 \mathrm{~km} \mathrm{~s}^{-1}$. It is not exceptional for Algol gainers to rotate faster than synchronously as shown by Koch et al. (1965). However, the difference being only $2.3 \sigma$, we cannot firmly conclude that a departure from synchronism exists in TZ Eri. On the other hand, $v \sin i$ may be overestimated, due to several factors. First, the rotational velocity is so large that we hardly reach the continuum of the correlation function, which can bias the fit. Second, the profile is fitted by a Gaussian, while the rotational profile itself does not 
Table 5. Orbital elements of the binary. For each component, the second line gives the estimated standard deviations of the parameters. A null uncertainty means that the corresponding parameter has been fixed before the convergence. The period has been fixed to the value it had in the middle of the time spanned by the $V_{\mathrm{r}}$ observations

\begin{tabular}{|r|r|r|r|r|r|r|r|r|r|r|}
\hline Star name & $\begin{array}{c}P \\
{[\text { days }]}\end{array}$ & $\begin{array}{r}T_{0} \text { [HJD } \\
-2450000]\end{array}$ & $e$ & $\begin{array}{r}V_{0} \\
{\left[\mathrm{~km} \mathrm{~s}^{-1}\right]}\end{array}$ & $\begin{array}{l}\omega_{1} \\
{\left[{ }^{\circ}\right]}\end{array}$ & $\begin{array}{c}K_{1,2} \\
{\left[\mathrm{~km} \mathrm{~s}^{-1}\right]}\end{array}$ & $\begin{array}{r}M_{1,2} \sin ^{3} i \\
{\left[M_{\odot}\right]}\end{array}$ & $\begin{array}{l}a_{1,2} \sin i \\
{\left[10^{6} \mathrm{~km}\right]}\end{array}$ & $N$ & $\begin{array}{c}(\mathrm{O}-\mathrm{C}) \\
{\left[\mathrm{km} \mathrm{s}^{-1}\right]}\end{array}$ \\
\hline TZ Eri A & 2.606132 & 83.4009 & 0.000 & -13.10 & 0.0 & 31.93 & 1.741 & 1.144 & 30 & 3.04 \\
& 0.0000000 & 0.0000 & 0.000 & 0.55 & 0.0 & 0.70 & 0.087 & 0.025 & \\
TZ Eri B & & & & & & 165.32 & 0.336 & 5.92 & 1 \\
& & & & & 3.73 & 0.017 & 0.13 & \\
\hline
\end{tabular}

Table 6. Characteristics of the 7 Geneva passbands (Rufener \& Nicolet 1988). $\lambda_{0}$ is the mean wavelength and $\mu$ is the secondorder moment (approximately half the passband width)

\begin{tabular}{|c|c|c|}
\hline Passband & $\lambda_{0}[\AA]$ & $\mu[\AA]$ \\
\hline$U$ & 3464 & 159 \\
\hline$B 1$ & 4015 & 188 \\
\hline$B$ & 4227 & 282 \\
\hline$B 2$ & 4476 & 163 \\
\hline$V 1$ & 5395 & 202 \\
\hline$V$ & 5488 & 296 \\
\hline$G$ & 5807 & 200 \\
\hline
\end{tabular}

Table 7. Adopted values of the logarithmic limb-darkening parameters which were kept fixed in the least-squares solution. For the primary, only the $y$ parameters were fixed (the fitted $x$ parameters are listed in Table 8 ). For the secondary, both $x$ and $y$ parameters had to be fixed

\begin{tabular}{lrrr}
\hline Passband & $y_{1}$ & $x_{2}$ & $y_{2}$ \\
\hline$U$ & 0.243 & 0.940 & -.515 \\
$B 1$ & 0.303 & 0.863 & -.240 \\
$B$ & 0.303 & 0.858 & -.240 \\
$B 2$ & 0.303 & 0.868 & -.240 \\
$V 1$ & 0.263 & 0.847 & -.043 \\
$V$ & 0.263 & 0.825 & -.043 \\
$G$ & 0.263 & 0.819 & -.043 \\
\hline
\end{tabular}

have this shape, even if it is convoluted by a Gaussian instrumental profile (the calibration was intended for slow rotators, with $v \sin i$ generally slower than $\sim 20 \mathrm{~km} \mathrm{~s}^{-1}$ ). Third, the calibration has been devised above all for cool, solar-type stars (although it does include a temperature term) and we apply it to a late A star, i.e. at the very border of its validity. Fourth, the $v \sin i$ determination is based on the hypothesis of a solar-type macroturbulence; this is probably not valid in a semi-detached short period system and, thus the value given in (5) could be an overestimate.

We did not attempt to derive the projected rotational velocity of the secondary component by using the corre- lation dip obtained with the NTT spectrum, because a new calibration would have been needed which is beyond the scope of this paper, and the correlation dip of the secondary is in any case very shallow.

It is interesting to notice that the "surface" or equivalent width of the CORAVEL autocorrelation dip of the primary is the same as for single late-A stars. We have $W=2.31 \pm 0.14 \mathrm{~km} \mathrm{~s}^{-1}$, while HD 2628, type A7III and $[\mathrm{Fe} / \mathrm{H}]=-0.02$, has $W=2.41$ and HD 110379, type F0V and $[\mathrm{Fe} / \mathrm{H}]=-0.07$, has $W=2.49$. This implies that the metallicity of TZ Eri is close to the solar value, in agreement with the result obtained in Sect. 4 on the basis of the photometric analysis.

\section{Photometric solution}

The photometric solution for TZ Eri eclipses was obtained with the Wilson-Devinney WD programme (Wilson \& Devinney 1971; Wilson 1992), using the version revised in 1995. We used the WD programme in MoDE 5, adapted for semi-detached systems, allowing a simultaneous computation on the seven Geneva photometric light curves, based on 393 measurements (see Sect. 3), and the radial velocity curves of both components (see Table 4).

Lobe filling of the cool loser component was assumed. The semi-major axis of the relative orbit was initially set to $A=10.2 R_{\odot}$ as calculated from radial velocity results (see Sect. 5). Orbital eccentricity is fixed to zero and longitude of the periastron to $90^{\circ}$. Primary star temperature was fixed to $7770 \mathrm{~K}$ as determined in Sect. 4. For both components, the stellar atmosphere models of Kurucz (1994) integrated by Nicolet (1998) through the Geneva photometry passbands (Rufener \& Nicolet 1988) have been used.

The bolometric albedos for hot and cool components were taken at the theoretical value of 1.0 and 0.5 respectively (radiative and convective cases). Noise was set to 1 because scintillation can been neglected, the photon noise being dominant (see Bartholdi et al. 1984). The grid resolution values were taken as 20, 20, 20, 20 for N1, N2, N1L and N2L respectively (see WD programme). For each of 


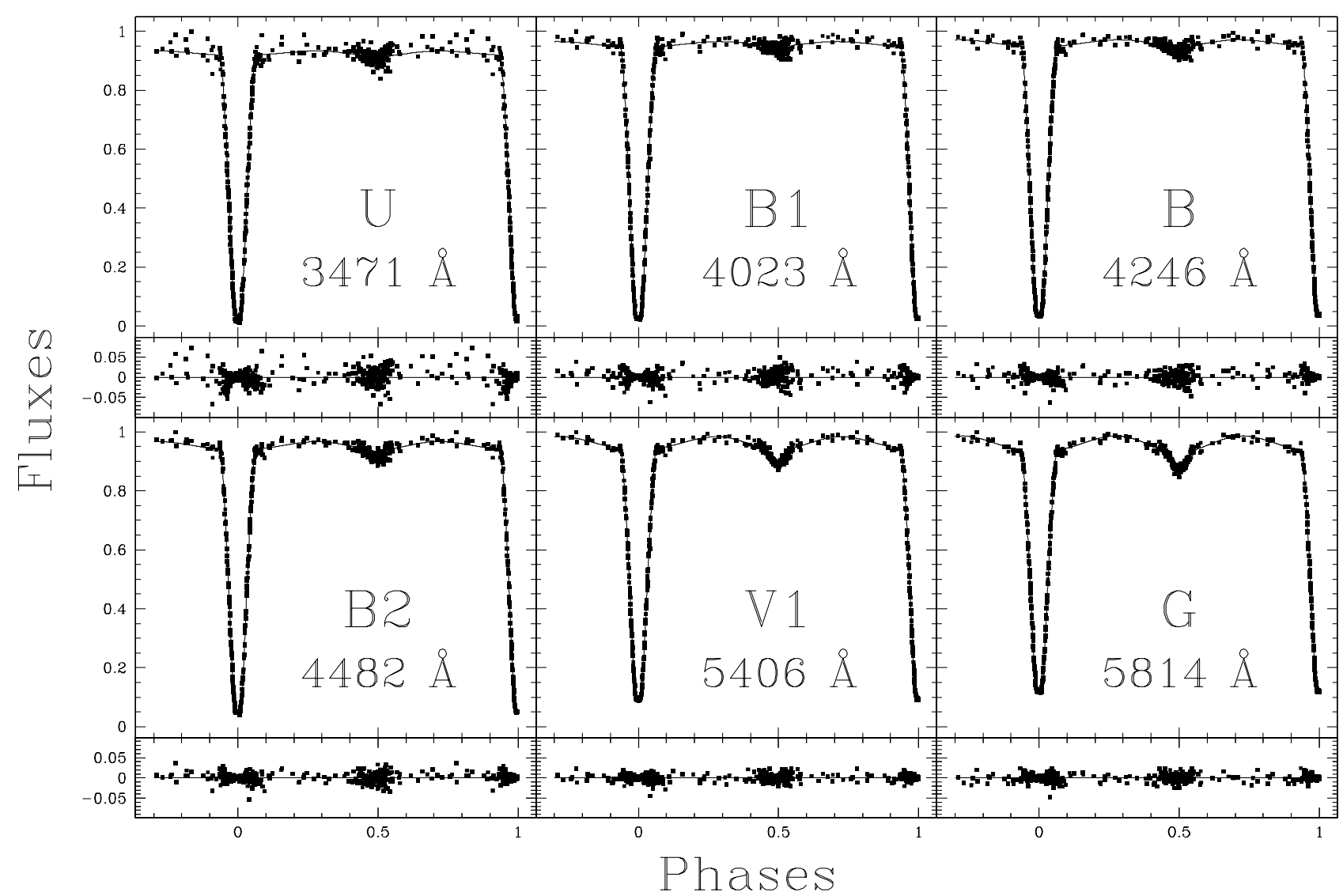

Fig. 4. Light curves of TZ Eri in 6 of the 7 Geneva photometric passbands $(U, B 1, B, B 2, V 1, G)$. The light curve in $V$ is given in Fig. 5

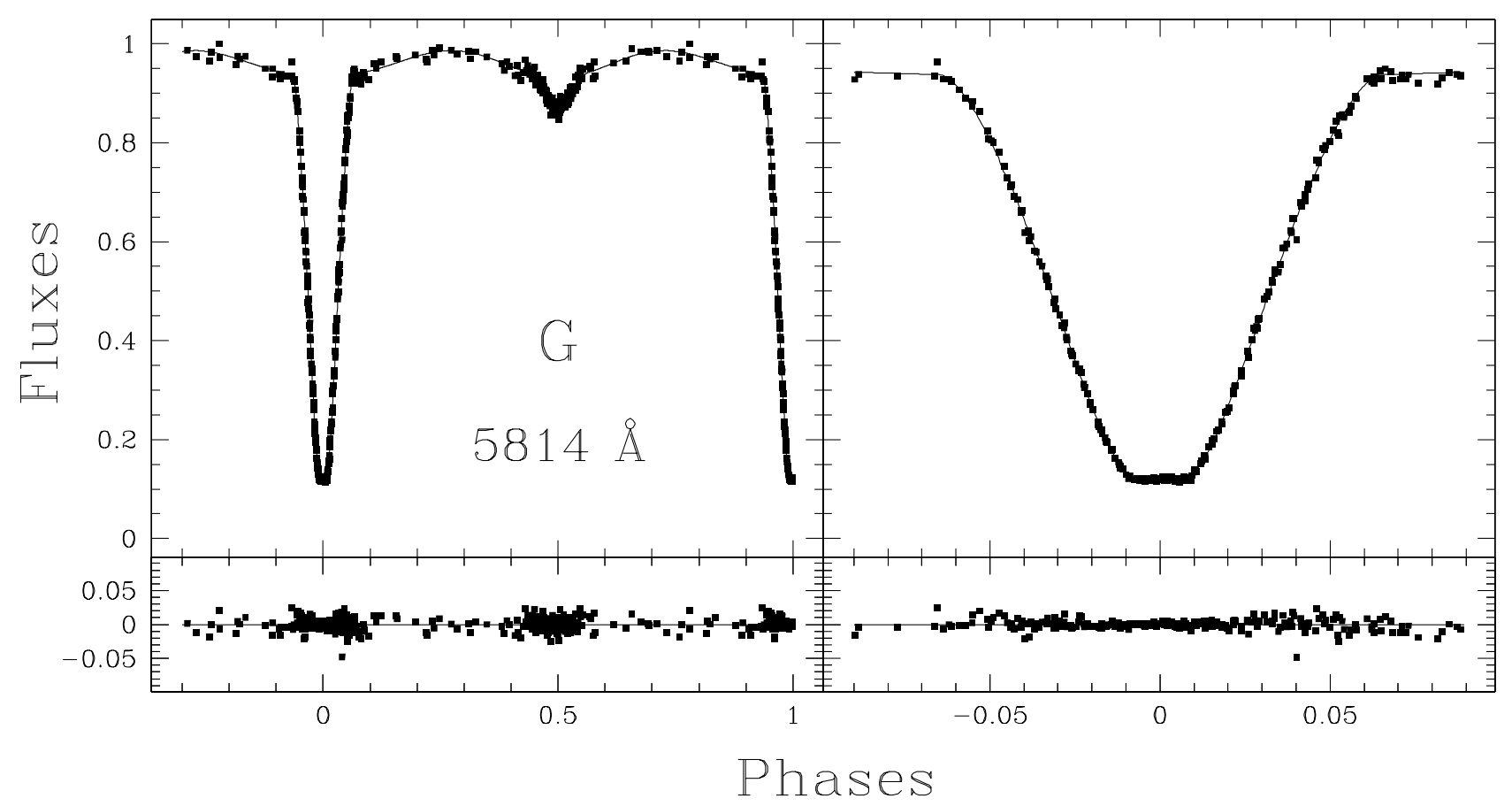

Fig. 5. The light curve of TZ Eri in magnitude $V$, with an enlargement of the primary eclipse 
Table 8. Adjusted parameters of the system TZ Eri from the Wilson-Devinney programme. The parameters are: the temperatures $T_{1}$ and $T_{2}$ of the primary (hot) and secondary (cool) components,

the semi-major axis $A$ of the relative orbit,

the orbital inclination $i$,

the mass ratio $q$ (the uncertainty assumes exact lobe filling for the secondary),

the potential of the surface of the primary component $\Omega_{1}$ (for the units, see Sect. 7 and WD programme),

the potential of the surface of the secondary component $\Omega_{2}$ (for the units, see Sect. 7 and WD programme), the exponent of the gravity darkening law $g_{2}$,

the normalized monochromatic luminosity in the seven Geneva passbands for the primary $L_{1} /\left(L_{1}+L_{2}\right)$, the normalized monochromatic luminosity in the seven Geneva passbands for the secondary $L_{2} /\left(L_{1}+L_{2}\right)$, the center-to-limb darkening factors for the primary $x_{1}$ in the seven passbands

\begin{tabular}{|c|c|c|c|c|c|c|}
\hline Parameters & $\begin{array}{l}\text { Values } \\
\left(T_{1}=7770 \mathrm{~K}\right)\end{array}$ & Uncertainties & $\begin{array}{l}\text { Values } \\
\left(T_{1}=7670 \mathrm{~K}\right)\end{array}$ & $\begin{array}{l}\text { Differences } \\
\text { " } 7670-7770 "\end{array}$ & $\begin{array}{l}\text { Values } \\
\left(T_{1}=7870 \mathrm{~K}\right)\end{array}$ & $\begin{array}{l}\text { Differences } \\
\text { "7870-7770" }\end{array}$ \\
\hline$T_{1}[\mathrm{~K}]$ & 7770 & & 7670 & -100 & 7870 & +100 \\
\hline$T_{2}[\mathrm{~K}]$ & 4563 & \pm 2 & 4614 & 51 & 4669 & 106 \\
\hline$A\left[R_{\odot}\right]$ & 10.57 & \pm 0.16 & 10.59 & 0.02 & 10.56 & -0.01 \\
\hline$i\left[^{\circ}\right]$ & 86.73 & \pm 0.03 & 86.77 & 0.04 & 86.71 & -0.02 \\
\hline$q=M_{2} / M_{1}$ & 0.1865 & \pm 0.0003 & 0.1862 & -0.0003 & 0.1867 & 0.0002 \\
\hline$\Omega_{1}$ & 6.46 & \pm 0.01 & 6.45 & -0.01 & 6.49 & 0.03 \\
\hline$\Omega_{2}$ & 2.20 & & 2.20 & 0.0 & 2.20 & 0.0 \\
\hline$g_{2}$ & 0.322 & & & & & \\
\hline$L_{1} /\left(L_{1}+L_{2}\right)$ & & & & & & \\
\hline$U$ & 0.9687 & \pm 0.0010 & 0.9692 & 0.0005 & 0.9692 & 0.0005 \\
\hline$B 1$ & 0.9644 & \pm 0.0003 & 0.9644 & 0.0 & 0.9644 & 0.0 \\
\hline$B$ & 0.9481 & \pm 0.0003 & 0.9481 & 0.0 & 0.9481 & 0.0 \\
\hline$B 2$ & 0.9312 & \pm 0.0003 & 0.9313 & 0.0001 & 0.9313 & 0.0001 \\
\hline$V 1$ & 0.8763 & \pm 0.0004 & 0.8765 & 0.0002 & 0.8765 & 0.0002 \\
\hline$V$ & 0.8676 & \pm 0.0005 & 0.8678 & 0.0002 & 0.8679 & 0.0003 \\
\hline$G$ & 0.8420 & \pm 0.0003 & 0.8422 & 0.0002 & 0.8421 & 0.0001 \\
\hline$L_{2} /\left(L_{1}+L_{2}\right)$ & & & & & & \\
\hline$U$ & 0.0313 & \pm 0.0023 & 0.0308 & -0.0005 & 0.0308 & -0.0005 \\
\hline$B 1$ & 0.0356 & \pm 0.0006 & 0.0356 & 0.0 & 0.0356 & 0.0 \\
\hline$B$ & 0.0519 & \pm 0.0005 & 0.0519 & 0.0 & 0.0519 & 0.0 \\
\hline$B 2$ & 0.0688 & \pm 0.0005 & 0.0687 & -0.0001 & 0.0687 & -0.0001 \\
\hline$V 1$ & 0.1237 & \pm 0.0006 & 0.1235 & -0.0002 & 0.1235 & -0.0002 \\
\hline$V$ & 0.1324 & \pm 0.0006 & 0.1322 & -0.0002 & 0.1321 & -0.0003 \\
\hline$G$ & 0.1580 & \pm 0.0004 & 0.1578 & -0.0002 & 0.1579 & -0.0001 \\
\hline \multirow[t]{7}{*}{$x_{1}$} & 0.655 & \pm 0.037 & \multirow{7}{*}{\multicolumn{4}{|c|}{ Determinant of normal equations: $1.2310^{-26}$}} \\
\hline & 0.770 & \pm 0.009 & & & & \\
\hline & 0.745 & \pm 0.009 & & & & \\
\hline & 0.735 & \pm 0.009 & & & & \\
\hline & 0.533 & \pm 0.013 & & & & \\
\hline & 0.542 & \pm 0.015 & & & & \\
\hline & 0.463 & \pm 0.012 & & & & \\
\hline
\end{tabular}

the seven Geneva magnitudes, the passband mean wavelength $\lambda_{0}$ was the one calculated by Rufener \& Nicolet (1988) as shown in Table 6 . We assigned the $\lambda_{0}$ value for the $B$ filter to the passband of the spectrographs used for the radial velocity measurements. Stellar rotation is assumed to be synchronized for both components. For both the primary and secondary components, a logarithmic limb-darkening law of the form:

$I=I_{0}(1-x+x \cos \theta-y \cos \theta \ln (\cos \theta))$

was assumed (Van Hamme 1993). For the secondary, both $x$ and $y$ parameters were fixed to their theoretical values, interpolated from Table 2 of Van Hamme (1993). Indeed, 
the secondary minimum is too shallow to allow the determination of $x_{2}$. For the primary, the $y_{1}$ parameter was fixed to its theoretical value, because it is not possible in practice to determine both $x_{1}$ and $y_{1}$ parameters since they are strongly correlated. The $x_{1}$ parameter, on the contrary, was left free and was fitted in the least-squares procedure, for each of the seven passbands. The adopted values of $y_{1}, x_{2}$ and $y_{2}$ are listed in Table 7 , while the fitted values of $x_{1}$ are given in Table 8 . The gravity darkening exponent $g_{2}$ is relevant to the secondary component and is defined by the expression (Wilson \& Biermann 1976):

$T_{\text {local }}=T_{\text {r.p. }}\left(\frac{a_{\text {local }}}{a_{\text {r.p. }}}\right)^{0.25 g}$

where $T_{\text {local }}$ is the local effective temperature, $T_{\text {r.p. }}$ is the temperature at a reference point which here is the component's pole, and $a_{\text {local }}, a_{\text {r.p. }}$ are the accelerations due to gravity at the same respective points.

The adjustable parameters are then semi-major axis $A$, inclination $i$, mass ratio $q=M_{2} / M_{1}$, cool star temperature $T_{2}$, hot and cool star luminosities $L_{1}$ and $L_{2}$ in each passband, cool star gravity darkening exponent $g_{2}$ (see Wilson \& Biermann 1976), hot star limb darkening coefficient $x_{1}$ in each passband and potential $\Omega_{1}$ at the hot star surface. In reality, $\Omega$ is a non-dimentional parameter which is a linear function of the true potential $\Psi$ (Kopal 1959; Wilson \& Devinney 1971). First, preliminary light curves were generated using the Light Curve sub-programme of WD until a satisfactory fit to the observed light curves was obtained. Second, the method recommended by Van Hamme \& Wilson (1986) was applied for the determination of the temperature and luminosity of the cool component. In a first step, $T_{2}$ was determined with IPB option (see the WD programme) equal to 0, i.e. the luminosity is coupled to the temperature. In a second step, temperature and luminosity were de-coupled $(I P B=1)$, and it was possible to determine $L_{2}$. Finally, the following sets of parameters were adjusted: first, successively $\left(T_{2}, \Omega_{1}, i\right),\left(L_{1}\right.$, $\left.L_{2}\right),(A, i),\left(x_{1}, g_{2}\right)$, and then simultaneously $\left(A, i, \Omega_{1}\right.$, $\left.q, L_{1}, L_{2}\right)$, as recommended by the users of the WD programme. The solution was carried out to the point where the probable errors of all these parameters were smaller than the computed parameter corrections.

Since the photometric temperature of the primary star is determined with an uncertainty of $\pm 100 \mathrm{~K}$ (see Sect. 4), it is interesting to calculate the solution also for the two $T_{1}$ values $7670 \mathrm{~K}$ and $7870 \mathrm{~K}$. The results are presented in Table 8 . The differences are very small, except of course on $T_{2}$.

It is especially noteworthy that, for $T_{1}=7770 \mathrm{~K}$, the value of $T_{2}(4563 \pm 2 \mathrm{~K})$ is very close to the value obtained in Sect. 4 on the basis of the photometric calibrations. The mass ratio $q=M_{2} / M_{1}=0.1865 \pm 0.0003$ in Table 8 is a little smaller than the value derived from the radial velocity analysis $\left(K_{1} / K_{2}=0.193 \pm 0.013\right.$, see Table 5$)$, but still well within the uncertainty of the spectroscopic determination. A similar difference can be noted on the semi-major axis of the relative orbit $A=a_{1}+a_{2}(10.16 \pm$ $0.22 R_{\odot}$ in Table 5 and $10.57 \pm 0.16 R_{\odot}$ in Table 8$)$. These differences are not surprising. We have to recall that the radial velocity curve for the secondary component is based on only one measurement. The constraints imposed by the photometric measurements of the eclipses produced an improvement of the orbital and physical parameters of TZ Eri.

The small difference between the photometric and spectroscopic values of $q$ could indicate that the lobe filling by the secondary is not complete. If this were true, the real value of the uncertainty on $q$ would be larger than the photometric value $(0.0003)$, may be as large as the spectroscopic value (0.013). Nevertheless, test solutions have been obtained with the cool star slightly detached from its Roche lobe, and the results are less satisfactory than those obtained in MoDE 5 of the WD code: as the cool star became farther detached from the lobe, solution errors grew. This confirms the semi-detached status of the system.

The uncertainties on the derived parameters are of three types:

- Intrinsic, i.e. resulting from the mathematical analysis of the light and radial velocity curves (e.g. $\pm 2 \mathrm{~K}$ on $T_{2}$, see Table 8). Note that the intrinsic errors on $R_{1,2}$ for $A$ fixed are an order of magnitude smaller than the values in Table 9.

- Strongly correlated with the determination of $A$. For example, in Table 9 , the errors on $M_{1,2}, R_{1,2}$ and $\log g_{1,2}$ almost entirely come from the uncertainty on $A$, and are pair wise strongly correlated (as well as with $A$ ).

- Depending on the photometric determination of $T_{\mathrm{eff}_{1}}$. For example, the values of $M_{\mathrm{bol}_{1,2}}$ strongly depend on the adopted value for $T_{\text {eff }_{1}}$.

The light curves for the seven filters $U, B_{1}, B, B_{2}, V_{1}$, $V$ and $G$ are shown in Figs. 4 and 5 . The quality of the fits is clearly extremely good. Figures 6 and 7 present two views of TZ Eri. Figure 6 is a "classical" representation of the two components in the equatorial plane. The 3dimentional representation of the potential (Fig. 7) allows a better understanding of the meaning of the Lagrange points and of the path for the flow of the material from the secondary when it fills its Roche lobe.

\section{Discussion}

\subsection{Limb-darkening}

Our adjusted values of $x_{1}$ (see Table 8) compare relatively well with the theoretical ones, in the sense that the difference hardly exceeds 0.1 , or $15-20 \%$ of the value (except for $V$ where the difference reaches 0.15$)$. Nevertheless, our values are systematically smaller than the theoretical ones (computed for a solar chemical composition). 
Table 9. Computed parameters of the system TZ Eri from the Wilson-Devinney programme. The parameters are, for the hot primary (1) and the cool secondary (2) components: the mass $M$, the mean radius $R$, the surface gravity $\log g$, the bolometric magnitude $M_{\mathrm{bol}}$ and the various radii (in units of semi-major axis) of the deformed components, i.e. pole (perpendicular to the orbital plane), point (in the direction of the other component), side (in the orbital plane, in the direction perpendicular to the direction of the other component) and back (in the direction opposite to the other component). The uncertainty on $M_{\text {bol }}$ is calculated with an uncertainty on $T_{\text {eff }}$ of $\pm 100 \mathrm{~K}$

\begin{tabular}{|c|c|c|c|}
\hline Parameters & $\begin{array}{l}\text { Values } \\
\left(T_{1}=\right. \\
\mathbf{7 7 7 0} \mathrm{K})\end{array}$ & $\begin{array}{l}\text { Values } \\
\left(T_{1}=\right. \\
\mathbf{7 6 7 0} \mathrm{K})\end{array}$ & $\begin{array}{l}\text { Values } \\
\left(T_{1}=\right. \\
\mathbf{7 8 7 0} \mathrm{K})\end{array}$ \\
\hline$M_{1}\left[M_{\odot}\right]$ & $1.97 \pm 0.06$ & 1.98 & 1.97 \\
\hline$M_{2}$ & $0.37 \pm 0.01$ & 0.37 & 0.37 \\
\hline$R_{1}\left[R_{\odot}\right]$ & $1.69 \pm 0.03$ & 1.69 & 1.68 \\
\hline$R_{2}$ & $2.60 \pm 0.04$ & 2.60 & 2.60 \\
\hline $\log g_{1}$ & $4.28 \pm 0.03$ & 4.28 & 4.28 \\
\hline $\log g_{2}$ & $3.17 \pm 0.03$ & 3.17 & 3.17 \\
\hline$M_{\mathrm{bol}_{1}}$ & $2.36 \pm 0.09$ & 2.41 & 2.32 \\
\hline$M_{\mathrm{bol}_{2}}$ & $3.74 \pm 0.13$ & 3.69 & 3.64 \\
\hline$r_{\text {pole }_{1}}[\mathrm{~A}]$ & $0.1592 \pm 0.0003$ & 0.1595 & 0.1585 \\
\hline$r_{\text {point }_{1}}$ & $0.1598 \pm 0.0003$ & 0.1601 & 0.1591 \\
\hline$r_{\text {side }_{1}}$ & $0.1596 \pm 0.0003$ & 0.1599 & 0.1589 \\
\hline$r_{\text {back }_{1}}$ & $0.1598 \pm 0.0003$ & 0.1601 & 0.1591 \\
\hline$r_{\text {pole }_{2}}[\mathrm{~A}]$ & $0.2282 \pm 0.0001$ & 0.2281 & 0.2283 \\
\hline$r_{\text {point }_{2}}$ & $0.34 \quad \pm 0.01$ & 0.3349 & 0.3351 \\
\hline$r_{\text {side }_{2}}$ & $0.2374 \pm 0.0001$ & 0.2373 & 0.2374 \\
\hline$r_{\text {back }_{2}}$ & $0.2696 \pm 0.0001$ & 0.2695 & 0.2697 \\
\hline
\end{tabular}

The discrepancies are larger than the formal errors indicated in Table 8 , which may partly, but probably not entirely be explained by the fact that the errors listed should represent only lower limits to the true ones.

Figure 8 illustrates the dependence of $x_{1}$ on wavelength for a theoretical star with $T_{\text {eff }}=7770 \mathrm{~K}$ and $\log g=4.28$, for the passbands of Strömgren's uvby system and Johnson's $U B V$ system (the values have been linearly interpolated in Table 2 of Van Hamme 1993). The fitted values for the Geneva $U, B 1, B, B 2, V 1, V, G$ passbands are shown for comparison.

The theoretical dependence of $x_{1}$ on wavelength satisfies very well the observations from the $U$ up to the $B 2$ passband, except for a small, systematic vertical shift. For the $V 1$ to $G$ passbands, however, the observed $x_{1}$ parameter is much smaller than the theoretical one. Since the error bars are not larger than the symbols in Fig. 8 (except marginally for $U$ ), there is undoubtedly a significant discrepancy which remains to be explained. We obtained a simultaneous solution in the seven passbands, thus at

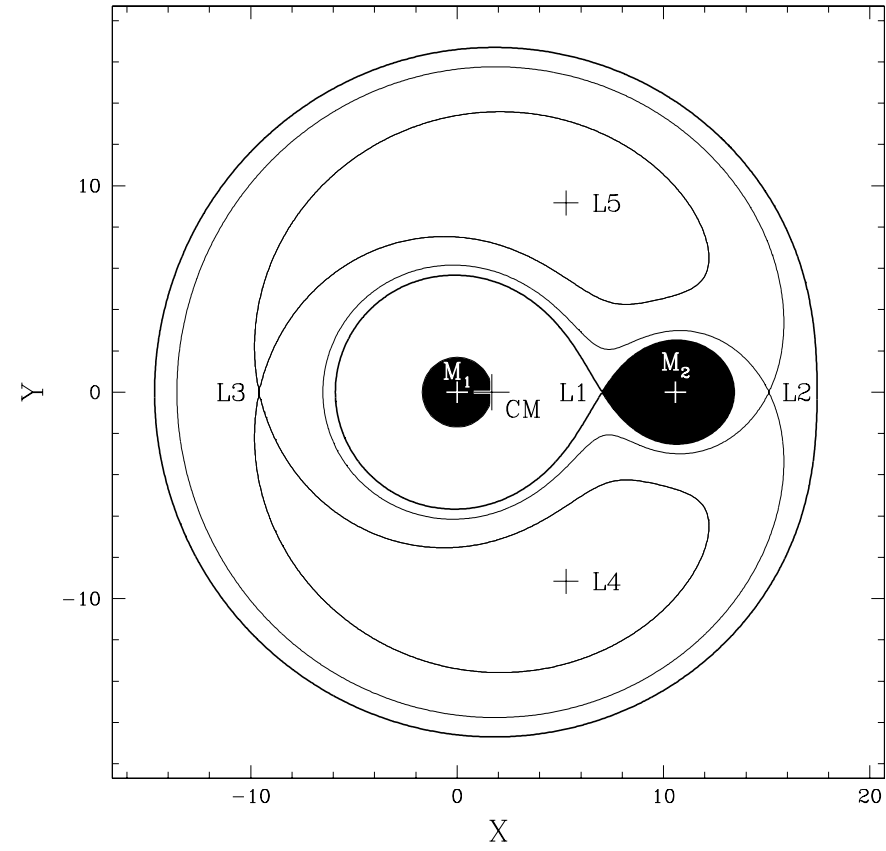

Fig. 6. Schematic view of the system in the equatorial plane (coordinates in $\left.R_{\odot}\right)$. Some equipotential lines and the Lagrange points are indicated. The secondary star fills its Roche lobe

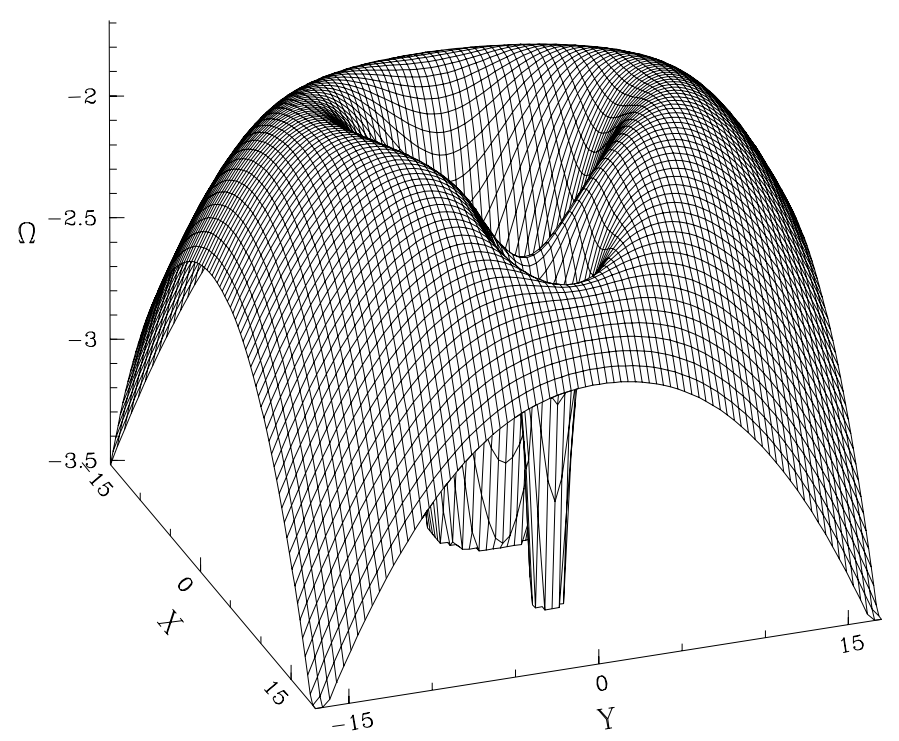

Fig. 7. Schematic view of the gravity potential $\Omega$ (see Sect. 7 for the definition) of the TZ Eri system. The horizontal coordinates are in $R_{\odot}$

least the relative values of $x_{1}$ should be reliable. But, even considering only differential values (e.g. $x_{1}(V)-x_{1}(B)$ ) a difference with the theoretical predictions does remain. Unfortunately, we did not see any recent discussion in the literature about the reliability of empirical limb-darkening coefficients in Algol-type systems. 


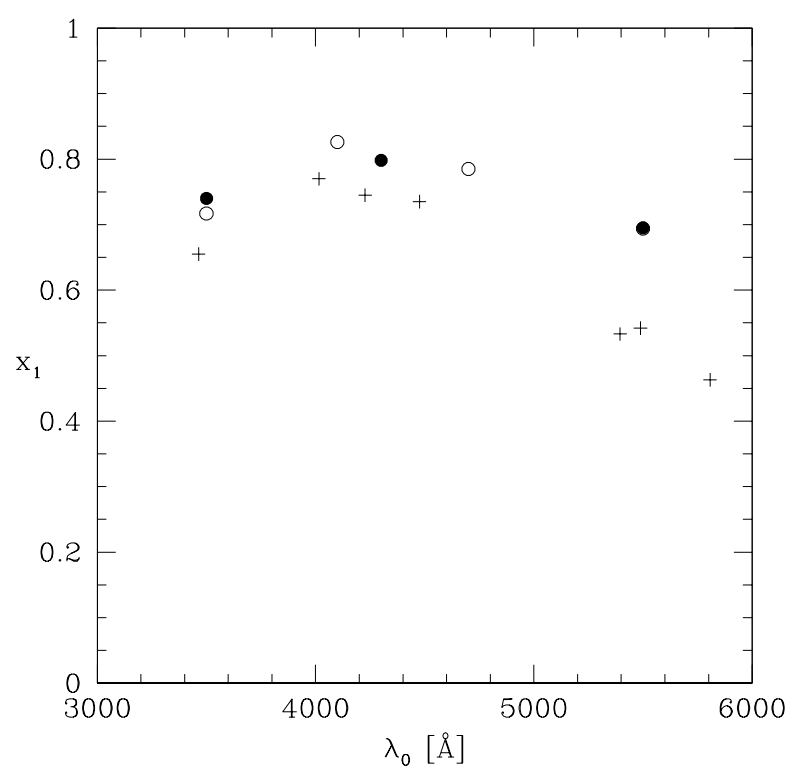

Fig. 8. Limb-darkening $x_{1}$ coefficient of the primary as a function of wavelength. Points are theoretical heterochromatic values for the uvby (open dots) and $U B V$ passbands (full dots), while crosses are for empirical values determined in this work for the passbands of the Geneva system. Notice that the theoretical points for the $v$ and the $V$ bands are superimposed

\subsection{Distance}

From the values of $V$ (Table 2$), M_{\mathrm{bol}}$ (Table 9$), E[B 2-V 1]$ and $[B 2-V 1]_{0}$ (Sect. 4 ), we derive a distance of $270 \pm 12$ pc for TZ Eri, by adopting a zero value for the bolometric correction $B C$ of the primary, according to the $B C$-colour relation of Flower (1977).

\subsection{Algol-type stars}

Sarma et al. (1996) gave a detailed discussion on the evolutionary status of Algol components, on the basis of a comparison of their global parameters (masses, radii, luminosities, temperatures) with those of normal stars. As these authors noted:

1. While primaries (mass gainers) of some semi-detached systems are overluminous, oversized and hotter for their masses, some others are underluminous, smaller and cooler.

2. Except for a few cases, the primaries are lying either on the main sequence or near it.

3. The secondaries (mass losers) have evolved off the main sequence (they were originally the more massive components).

Figures 9 and 10 present the Mass-Luminosity diagram $(\log L$ vs. $\log M)$ and the HR diagram $(\log L$ vs. $\log T$ ) for the Algol systems in the lists by Sarma et al. (1996) and Maxted \& Hilditch (1996) having a mass of the primary smaller than $4 M_{\odot}$. The 3 systems with primaries off the main sequence (RZ Cnc, AR Mon, AW Peg) are not represented. The underluminous (black dots) and overluminous (black squares) have been separated in Fig. 10 by using the ZAMS (Schaller et al. 1992) to make the separation. We can see that:

1. TZ Eri belongs to the group of the underluminous primaries.

2. The mass of TZ Eri's primary is the smallest of the group of these underluminous primaries.

3. The luminosity of TZ Eri's primary is that of a main sequence star of $1.70 M_{\odot}$ located near the ZAMS (see Fig. 10), while the determined mass is $1.98 M_{\odot}$ (see Table 9).

4. With respect to a main sequence star of the same mass, TZ Eri's primary is underluminous by $\sim 0.3$ in $\log L$ (or fainter by $\sim 0.75$ in $M_{\text {bol }}$ ) and cooler by $\sim 900 \mathrm{~K}$ in $T_{\text {eff }}$.

5. The mass transfer from the secondary to the primary has been important, taking into account the present masses of the components, i.e. $0.37 M_{\odot}$ and $1.98 M_{\odot}$. The minimum value of this transfered mass is given by:

$$
\Delta M=M_{1}-\left(M_{1}+M_{2}\right) / 2 \simeq 0.80 M_{\odot} .
$$

\section{Conclusion}

The simultaneous adjustment of the light curve model on high precision photometric data in seven passbands, ranging from 3400 to $6000 \AA$, put strong constraints on the physical and orbital parameters of TZ Eri. This is our first analysis of an Algol system on the basis of measurements made in the Geneva photometric system. The essential results are presented in Figs. 4 and 5, Tables 8 and 9, and in the Abstract. The quality of the fits on the light curves and the uncertainties on the parameters show that this analysis has been successful.

A further improvement on the determination of the physical parameters of the components of TZ Eri, in particular the mass ratio, would result from the acquisition of additional high $\mathrm{S} / \mathrm{N}$ spectra of the secondary cool component. Indeed, the present study is based on only one spectrum of the secondary and this is not completely satisfying. However, this spectrum was obtained using the $3.5 \mathrm{~m}$ NTT telescope at La Silla and we were extremely lucky to have access to this large telescope for this single measurement.

According to Maxted \& Hilditch (1996), the number of Algol-type binary systems for which the absolute parameters are determined on the basis of a self-consistent solution of both the light and radial velocity curves is very limited. They mentioned only 9 systems for which masses, radii and luminosities are known to accuracies typically better than $5 \%$. In this context, it is important to enlarge the sample of very well known Algols, and this was the aim of our study on TZ Eri. 


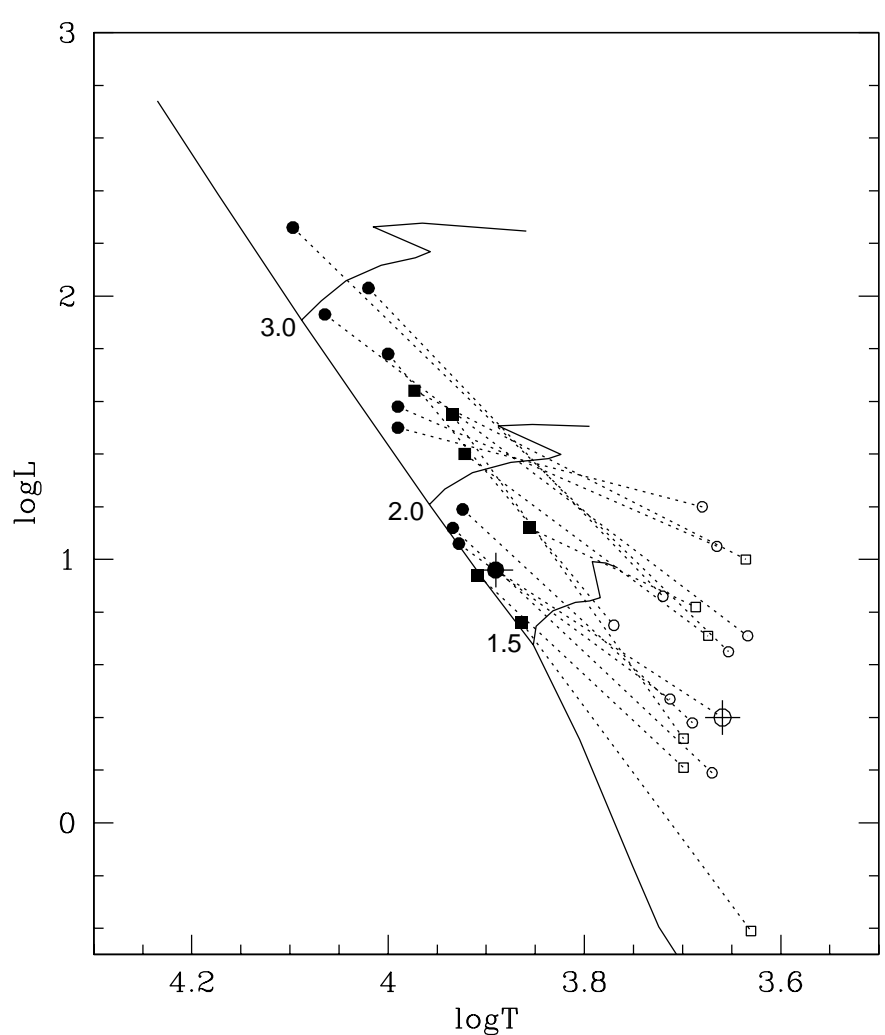

Fig. 9. Theoretical HR diagram for the components of Algol systems with very precisely determined physical parameters according to the lists by Sarma et al. (1996) and Maxted \& Hilditch (1996), and to this paper for TZ Eri (see Sect. 8.3). Primaries are identified with filled symbols and secondaries with open symbols. TZ Eri is identified with plus symbols. Dots and squares refer to the systems having respectively underand overluminous primaries with respect to their masses (see Fig. 10). The Zero-Age Main Sequence and 3 evolutionary tracks $\left(3.0,2.0\right.$ and $\left.1.5 M_{\odot}\right)$ are drawn, according to Schaller et al. (1992)

Acknowledgements. This work has been initiated by our late colleague Zdenek Kviz who obtained himself the major part of the photometric data; indeed, we would have preferred to do this analysis with him. We are grateful to Drs. R.E. Wilson for the programme of analysis of the light curves, B. Nicolet for the treatment of the Kurucz's fluxes, D. Raboud for the NTT observations of the secondary, J.-C. Mermilliod and J. Andersen for the CORAVEL measurements, M. Künzli for the photometric observations of 1996 and M. Studer for the reduction of the NTT spectrum. All graphs have been produced using the SuperMongo package (Lupton \& Monger 1998). This work has been partly supported by the Swiss National Science Foundation. The Mount Laguna Observatory is operated jointly by San Diego State University and the University of Illinois.

\section{References}

Baranne A., Mayor M., Poncet J.L., 1979, Vistas Astron. 23, 279

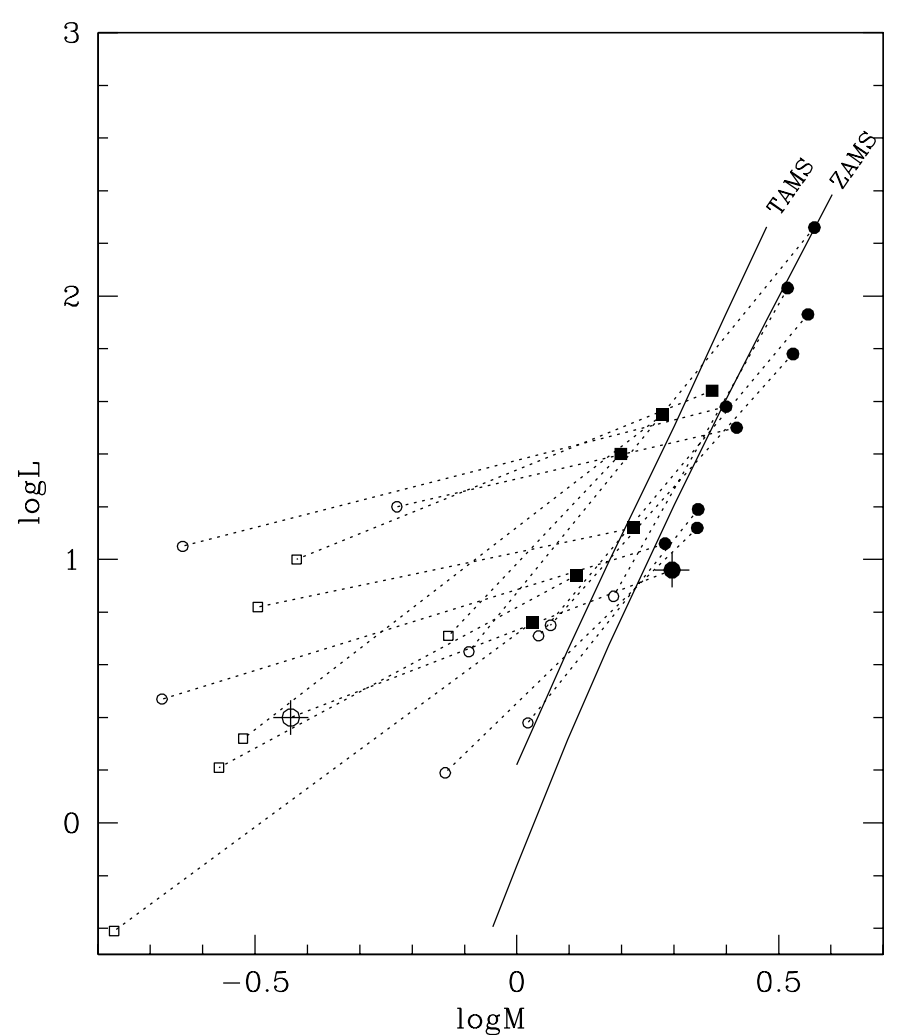

Fig. 10. Mass-Luminosity relation for the components of the same Algol systems as those in Fig. 9. The zero-age main sequence (ZAMS) and the Terminal-Age Main Sequence (TAMS) are drawn, according to Schaller et al. (1992). Primaries are identified with filled symbols and secondaries with open symbols. TZ Eri is identified with plus symbols. Dots and squares refer to the systems having respectively under- and overluminous primaries with respect to the ZAMS

Bartholdi P., Burnet M., Rufener F., 1984, A\&A 134, 290

Benz W., Mayor M., 1984, A\&A 138, 183

Brancewicz H.K., Dworak T.Z., 1980, Acta Astron. 30, 501

Burki G., Kviz Z., North P., 1992, A\&A 256, 463

Burnet M., Rufener F., 1979, A\&A 74, 54

Cannon A.J., 1934, Harvard College Obs. Bull. 897, 12

Etzel P.B., 1989 (private communication to Z. Kviz)

Flower P.J., 1977, A\&A 54, 31

Golay M., 1980, Vistas Astron. 24, 141

Golay M., Peytremann E., Maeder A., 1969, Publ. Obs. Genève, Série A 76, 44

Hauck B., 1994, ASP Conf. Ser. 60, 157

Kaitchuck R.H., Park E.A., 1988, ApJ 325, 225

Kaitchuck R.H., Honeycutt R.K., 1982, PASP 94, 532

Kholopov P.N., Samus N.N., Frolov M.S., et al., 1985, General Catalogue of Variable Stars. Nauka Publ. House, Moscow

Koch R.H., Olson E.C., Yoss K.M., 1965, ApJ 141, 955

Kopal Z., 1959, Close Binary Systems. Chapman \& Hall Ltd., London, p. 127

Künzli M., North P., Kurucz R.L., Nicolet B., 1997, A\&AS 122,51

Kurucz R.L., 1994, CD-ROM 19 (Solar abundance atmosphere models for $0,1,2,4,8 \mathrm{~km} \mathrm{~s}^{-1}$ )

Locher K., 1997, Bedeckungsveränderlichen Beobachter der Schweizerischen Astronomischen Gesellschaft (Swiss 
Astronomical Society's Eclipsing Variable Observers) BBSAG No. 114, p. 5

Lupton R.H., Monger P., 1998, SuperMongo Graphics and Computational Package. Princeton Astronomical Observatory and MacMaster University

Manfroid J., Renson P., Catalano F.A., et al., 1991, A\&AS 90, 19

Maxted P.F.L., Hilditch R.W., 1996, A\&A 311, 567

Nicolet B., 1981a, A\&A 97, 85

Nicolet B., 1981b, A\&A 104, 185

Nicolet B., 1994, ASP Conf. Ser. 60, 182

Nicolet B., 1998 (private communication)

Olson E.C., Etzel P.B., 1993, AJ 106, 342

Renson P., 1978, A\&A 63, 125

Rufener F., 1988, Catalogue of Stars Measured in the Geneva
Observatory Photometric System, $4^{\mathrm{e}}$ Édition, Obs. Genève Rufener F., Nicolet B., 1988, A\&A 206, 357

Sarma M.B.K., Vivekananda Rao P., Abhyankar K.D., 1996, ApJ 458, 371

Schaller G., Schaerer D., Meynet G., Maeder A., 1992, A\&AS 96, 269

Van Hamme W., 1993, AJ 106, 2096

Van Hamme W., Wilson R.E., 1986, AJ 92, 1168

Wilson R.E., 1992, Documentation of Eclipsing Binary Computer Model, University of Florida, Gainesville

Wilson R.E., Devinney E.J., 1971, ApJ 166, 605

Wilson R.E., Biermann, 1976, A\&A 48, 349

Yoon T.S., Honeycutt R.K., Kaitchuck R.H., Schlegel E.M., 1994, PASP 106, 243 